\title{
Corneal Wavefront Aberrations in Patients Wearing Multifocal Soft Contact Lenses for Myopia Control
}

\author{
Frank Spors $^{1,2^{*}}$, Donald J. Egan ${ }^{1,2}$, Jie Shen ${ }^{1,2}$, Lance E. McNaughton ${ }^{1,2}$, Stuart Mann ${ }^{1,2}$, Neil M. Patel ${ }^{1}$ \\ ${ }^{1}$ Western University of Health Sciences, Pomona, USA; ${ }^{2}$ College of Optometry, Pomona, USA. \\ Email: *fspors@westernu.edu
}

Received March 11 ${ }^{\text {th }}, 2012$; revised April 20 ${ }^{\text {th }}, 2012$; accepted May $14^{\text {th }}, 2012$

\begin{abstract}
Purpose: The purpose of this study was to evaluate the change in corneal wavefront aberrations in young adults who have been fit with multifocal soft contact lenses for myopia progression control. Findings have been analyzed for statistical significance and clinical relevance and compared to reportedly successful Orthokeratology outcomes. Methods: The dominant eye of 40 participants ( 27 women, 13 men; mean age $27.3 \pm 3.2$ years; range 23 to 39 years) was fit with Proclear Multifocal center-distance lenses (Coopervision, Pleasanton, USA) having a variety of distance powers and reading additions. Refractive errors were limited to a range of $-6.00 \mathrm{D}$ up to $+1.00 \mathrm{D}$ of sphere, and no greater than $-1.00 \mathrm{D}$ of cylinder. Corneal wavefront measurements were performed over $6 \mathrm{~mm}$ diameters with a Zeiss Atlas 9000 corneal topographer (Zeiss Meditec, Dublin, USA) prior to, and following lens fitting. Data were converted into rectangular Fourier optics terms $M, J_{0}, J_{45}$ and RMS values for each reading addition were statistically analyzed. Following evaluation of statistical significance and clinical relevance, results were compared to published data from successful Orthokeratology treatments. Results: Statistically significant changes in higher order aberrations were detected for lenses of all reading additions. Lens groups with higher Add powers demonstrated stronger changes with increased significance. Final RMS values relating to 2nd, 3rd and 4th Zernike orders reached clinical significance with a wavefront error of $0.10 \mu \mathrm{m}$, the equivalent of $0.25 \mathrm{D}$. Moreover, as Add powers increased, 3rd and 4th order aberrations likewise showed an increase. Pre-fitting astigmatism values accounted for the highest recorded aberrations and remained predominantly unchanged. Conclusion: Proclear Multifocal center-distance contact lenses were found to increase higher order wavefront aberrations in a manner dependent on their Add power. In comparison to successful Orthokeratology outcomes, the amounts of resulting aberrations are notably different.
\end{abstract}

Keywords: Multifocal Soft Contact Lenses; Wavefront Aberrations; Myopia; Myopia Progression Control; Orthokeratology; Zernike Polynomials

\section{Introduction}

Myopia is one of the most common ocular anomalies in the world [1]. Recent epidemiology studies cite a growing incidence of myopia that includes a heightened level of severity $[2,3]$. The prevalence of myopia is estimated to be $25 \%$ of adults in the United States [4]. In Taiwan, Hong Kong, and Singapore however, the prevalence of myopia in young adults is estimated at up to $60 \%$ to $80 \%$ [5]. The Beaver Dam Report on longitudinal refractive error changes in approximately 5000 people in the city and township of Beaver Dam, Wisconsin, US, over a 10year period showed an increase in the prevalence of myopia and even a possible increase in the prevalence and severity of high myopia [6,7]. Due to the growth in technology and knowledge within the fields of genetics, studies have been conducted to identify genetic loci as-

${ }^{*}$ Corresponding author. sociated with various conditions, including myopia. To date, an explanation for the onset and progression of simple myopia is given by both genetic susceptibility, especially associated with the PAX6 gene, and a conducing environment $[8,9]$. However, the exact causes for myopia progression are still unknown and under investigation. A strong impact on the onset and progression of myopia can be assigned to the retinal image quality [10]. Studies by Hung et al. in 2005 on infant monkeys demonstrated that the peripheral retina influences eye growth and refractive development and subsequently drives the emmetropization process after birth. The image quality at the central retina, the fovea, did not have an impact. While form deprivation in the retinal periphery accelerated elongation of the eye, resulting in myopia, even complete elimination of the central fovea with LASER ablation did not change the regular growth pattern [10]. Based on the convincing evidence provided by the Hung 
et al. study, peripheral retinal image quality in humans is a matter of great interest that remains open to further investigation. Here we have to differentiate between lower order and higher order aberrations which can be described by Zernike polynomials [11]. Zernike polynomials form a complete set of functions that are orthogonal over a circle of unit radius and serve as a set of basic functions. This property therefore renders them suitable for accurately describing wavefront aberrations. They are usually expressed in polar coordinates, and are readily convertible to Cartesian coordinates. These polynomials are mutually orthogonal, and are therefore mathematically independent. They can be scaled which allows a meaningful relative comparison between them. A common way to depict them is the so called Zernike pyramid as shown in Figure 1. In order to utilize Zernike polynomials for wavefront analysis and to assess their impact on the visual function, lower and higher orders become of interest. Relevant lower order aberrations include sphere and cylinder. Little is known about the impact of the lower order aberration cylinder and all higher order aberrations on myopia progression. Numerous reports exist about the impact of defocus, which equals the lower order aberration sphere. While emmetropic and hyperopic individuals tend to have a myopic defocus at their peripheral retina, myopic individuals have a relative hyperopic periphery [12]. A hyperopic defocus in the peripheral retina is likely to be a good stimulus for the onset and progression of myopia, independent of the central focus quality. This hyperopic defocus is associated with a prolate retinal shape, which has been found to be dominant in myopes [13]. Such findings are particularly remarkable since traditional tests used for determining visual function, degree of ametropia and optical defocus only assess the central retinal region. We can assume that a key element to compensate for already existing amounts of myopia and to inhibit further progression of myopia is a specific retinal image profile. In order to allow good visual acuity, this profile cannot be defocused in the central retina. At the same time, in order to inhibit myopia progression the image has to be myopically defocused or emmetropic in the peripheral retina. Figure 2 depicts this relationship. Historically, generating such a profile has been a challenge for the broad variety of optical treatment options and refractive surgery procedures that are currently available. A common routine of compensating myopic refractive error is the use of spectacle lenses with negative refractive power. Interestingly, all single vision spectacle lenses induce peripheral hyperopic defocus. The magnitude of this effect escalates with increasing refractive error and eccentricity [14]. In this manner it is clear that the desired retinal image profile is not produced. Hence, while the standard approach to refractive error correction is good for compensating central defocus by allowing good central visual acuities, its utility for myopia progression control would seem to be largely unmet. Other non-surgical treatment options for vision correction include unifocal contact lenses, Orthokeratology,

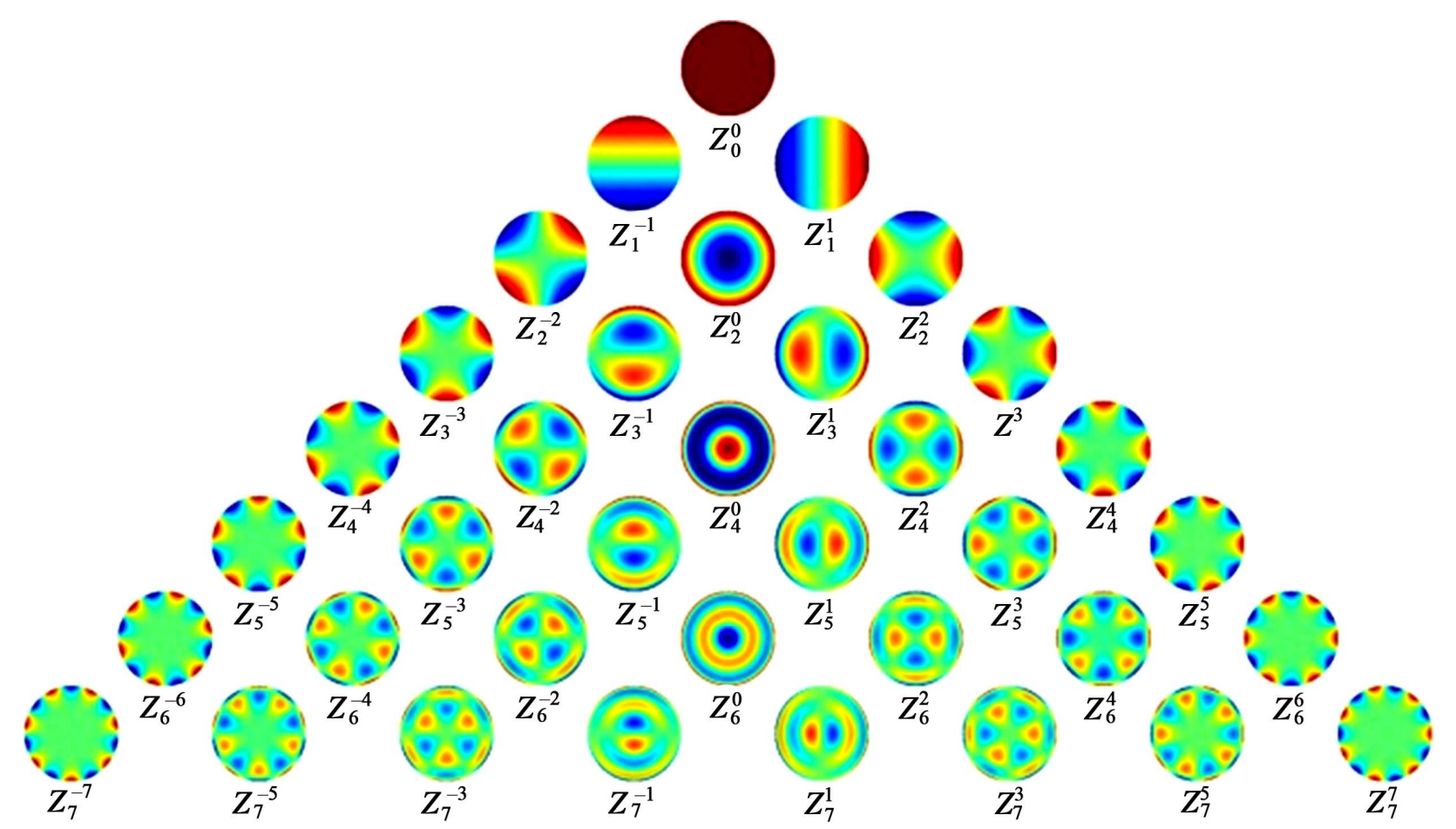

Figure 1. Zernike pyramid displaying individual color coded wavefront errors organized by Zernike coefficients up to the 7th order. The Zernike notation contains the coefficient $(Z)$, the order (subscript) and the radial frequency (superscript). 


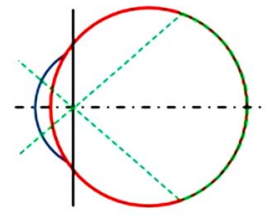

Spherical retinal shape $\Rightarrow$ no peripheral defocus

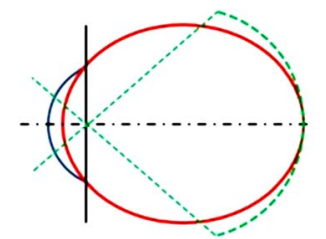

Prolate retinal shape $\Rightarrow$ hyperopic peripheral defocus

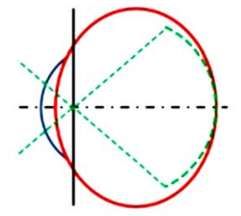

Oblate retinal shape $\Rightarrow$ myopic peripheral defocus

Figure 2. Relationship of different retinal shapes and peripheral retinal image quality (defocus) when the central refractive error is zero for all displayed schematic eyes (chief rays cross at the nodal points).

multifocal contact lenses, and spectacle lenses with specifically designed multifocal free-form surfaces [15-17]. All of these systems have a varying potential to induce peripheral myopic defocus and therefore may be beneficial for myopia progression control. Orthokeratology alters the corneal front surface in a way such that the resultant optics produces a focused image at the central retina and the desired myopic defocus at the peripheral retina $[18,19]$. Regular soft contact lenses have the potential to induce peripheral myopic defocus only if their power exceeds $-6.00 \mathrm{D}[20]$. Hence their value for myopia progression control is very limited, since this pattern ideally should start in the early phases of myopia development. Multifocal contact lenses with a concentric power structure such that the central portion contains the distance power and the mid-peripheral portion carries the near power provide a desired optical pattern. Originally introduced for presbyopic patients, their use for myopia progression control in young adults is an interesting application and expansion of the fitting range. If soft contact lenses are being used, their large diameter results in relatively little movement during the blink cycle, so that the lens optics stay aligned to the visual axis. This alignment is desirable for a stable retinal image. The front surface structures of such multifocal contact lenses have the theoretical ability to mimic the altered anterior corneal surfaces in Orthokeratology. Hence, the optics of both systems should have comparable characteristics. Accordingly, these lenses have been proposed as treatment option for myopia control $[21,22]$. A similar principle has been proposed for specifically designed spectacle lenses. Utilization of free form technology enables manufacturing of concentric lens surfaces with increasing refractive power toward the periphery. However in contrast to a multifocal contact lens such a spectacle lens is not in contact with the patient's eye and it does not follow the gaze as a contact lens does. Therefore, the desired optical effect is only present for one particular gaze position, which limits the treatment effect substantially [16]. Of the aforementioned non-surgical options, to date, the two systems that haven shown significant potential to control myopia progression are Orthokeratology and concentric multifocal center-distance contact lenses [23-
25].

Orthokeratology, unlike unifocal spectacle lenses and contact lenses, induces a characteristic higher order wavefront aberration pattern which by itself may contribute to the effect of controlling myopia progression [26]. If instead, multifocal soft contact lenses are being used for the same purpose, the resulting higher order wavefront aberration patterns are expected to be comparable. However, where the rigid back surfaces of Orthokeratology lenses induce evenly flattened centered corneal areas; spherical soft lenses conform to the corneal shape and do not neutralize any significant amount of astigmatism. That usually limits their use in astigmats since most multifocal frequent replacement contact lenses are only available in sphere power. In clinical practice for example, these lenses are usually fit on patients manifesting usually no more than $1.00 \mathrm{D}$ of refractive astigmatism. Nevertheless, with the prevalence of astigmatism among the US population being estimated to be $36 \%$, the likelihood that multifocal contact lenses, when used for myopia control, are being fit on astigmatic patients is relatively high [27].

In further consideration of these two treatment modes from a more technical standpoint, the central optical zone of center distance multifocal lenses is approximately 1.5 $\mathrm{mm}-2.5 \mathrm{~mm}$ smaller than the corneal treatment zone in Orthokeratology (Figure 3). In relation to the patient's entrance pupil this likely results in different amounts of higher order wavefront aberrations. The question is whether or not these differences are statistically and clinically relevant.

In our study we measured the changes in wavefront aberrations generated by concentric center-distance multifocal soft contact lenses from different Add power groups and analyzed the statistical significance and clinical relevance of these changes. Finally, we compared the changes with those that have been well documented in patients undergoing Orthokeratology [28]. All higher order aberrations were evaluated. In addition, special emphasis was put on lower order residual astigmatism, which, in contrast to Orthokeratology, cannot be fully compensated for with the frequent replacement contact lenses used in this study. 

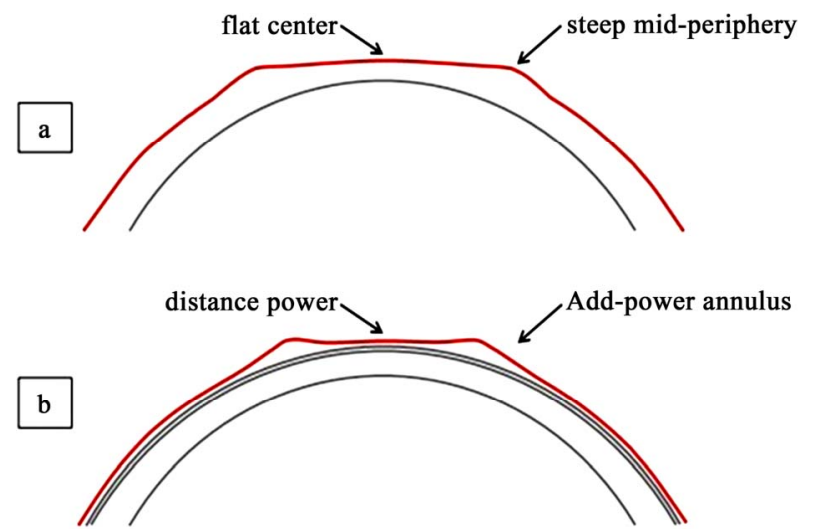

Figure 3. Cross sections of corneas for comparison of Orthokeratology (a), and a multifocal soft contact lens with center-distance design (b). Local changes in surface radii are displayed exaggerated and the optically relevant surfaces are drawn in red color. Flat surface radii are associated with low focal power, and steep surface radii are associated with high focal power.

\section{Objectives}

The purpose of this study was to analyze the remaining amount of astigmatism (2nd Zernike order) and higher order wavefront aberrations (3rd to 7th Zernike order) in young adults wearing concentric multifocal center-distance soft contact lenses with different Add powers. Since local changes in refractive power for these contact lenses, as well as for the patients' corneas, are solely based on local changes of front surface radii, these measurements can be done with a corneal topographer. The analyses were based on pre/post topographical measurements. RMS values of grouped wavefront aberrations were evaluated. The statistical significance and clinical relevance of these errors was further evaluated.

\section{Methods}

The dominant eyes of 40 participants were fit with varying powers of Proclear Multifocal D lenses (Coopervision, Pleasanton, USA). Corneal wavefront analysis was performed for simulated $6 \mathrm{~mm}$ entrance pupil diameters pre and post lens fit with the Zeiss Atlas 9000 corneal topographer (Zeiss Meditec, Dublin, USA). The sphere power of each selected lens was equal to the spherical component of the individual distance refraction. Four different Add powers $(+1.00 \mathrm{D},+1.50 \mathrm{D},+2.00 \mathrm{D},+2.50$ D) were fit on each patient. Fittings were evaluated 20 min after lens insertion following confirmation of good fit and centration.

The research followed the tenets of the Declaration of Helsinki and was approved by the IRB of Western University of Health Sciences (\#11/IRB/073). All measurements were conducted at the Health Education Center at Western University of Health Sciences.

\subsection{Subjects and Inclusion Criteria}

Forty University students ( 27 women, 13 men) with ages from 23 to 39 years $(27.3 \pm 3.2$ years $)$ were recruited for this study. Refractive errors were limited to the recommended fitting range of the tested lenses which includes $-6.00 \mathrm{D}$ up to $+1.00 \mathrm{D}$, and no greater than $-1.00 \mathrm{D}$ of cylinder. Expressed in rectangular Fourier optics terms, the mean spherical equivalent $M$ was $-2.69 \mathrm{D}$ (SD 2.08 $\mathrm{D}), J_{0}$ was $0.00 \mathrm{D}(\mathrm{SD} 0.16 \mathrm{D})$, and $J_{45}$ was $0.01 \mathrm{D}$ (SD $0.13 \mathrm{D})$. Participants did not suffer from any eye disease or injury and were not taking any ocular or systemic medications.

\subsection{Contact Lenses}

Proclear Multifocal D lenses have a concentric progressive front curve design. The distance power is in the lens center and spreads over a diameter of $2.3 \mathrm{~mm}$, followed by an annular zone which is progressively increases in focal power toward its periphery. The outermost optically relevant region is the near zone which extends to a total diameter of $8.5 \mathrm{~mm}$ (Figure 4). The lens material, omafilcon A, has a water content of $62 \%$. Selected lenses had a distance refractive power ranging from $-6.00 \mathrm{D}$ to $+1.00 \mathrm{D}$, and Add powers of $+1.00 \mathrm{D},+1.50 \mathrm{D},+2.00 \mathrm{D}$, and $+2.50 \mathrm{D}$. All lenses had a base curve radius of 8.70 $\mathrm{mm}$ and a diameter of $14.4 \mathrm{~mm}$. These contact lenses are recommended for replacement on a monthly basis.

\subsection{Topographical Measurements and Wavefront Analysis}

Topographical measurements were based on Placido ring reflex image analysis and were done with the Zeiss Atlas 9000 corneal topographer, an accurate and widely used clinical instrument [29]. Wavefront analysis was performed before and after each lens fitting for each Add power across the participants' pupils, and limited to diameters of $6 \mathrm{~mm}$ in order to have statistically comparable data. Wavefront distortions have been displayed and analyzed in $\mu \mathrm{m}$ as shown in Figure 5. For further statistical evaluation, data sets have been expressed in Zernike coefficients up to the 7th order. Corneal topographers capture images of illuminated Placido rings produced by the first optical surface in the pathway of light. By analyzing distortions of these images, wavefront aberrations can be calculated, displayed as color coded wavefront maps,

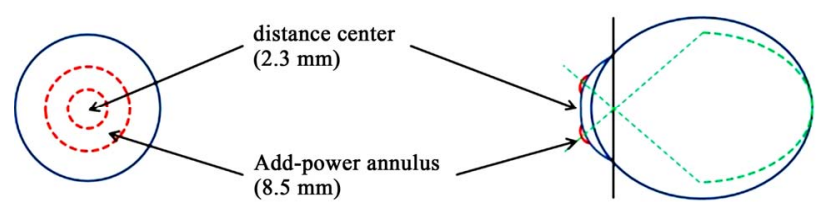

Figure 4. Optically relevant zones of a Proclear Multifocal $D$ lens and their effects on the retinal image structure. 


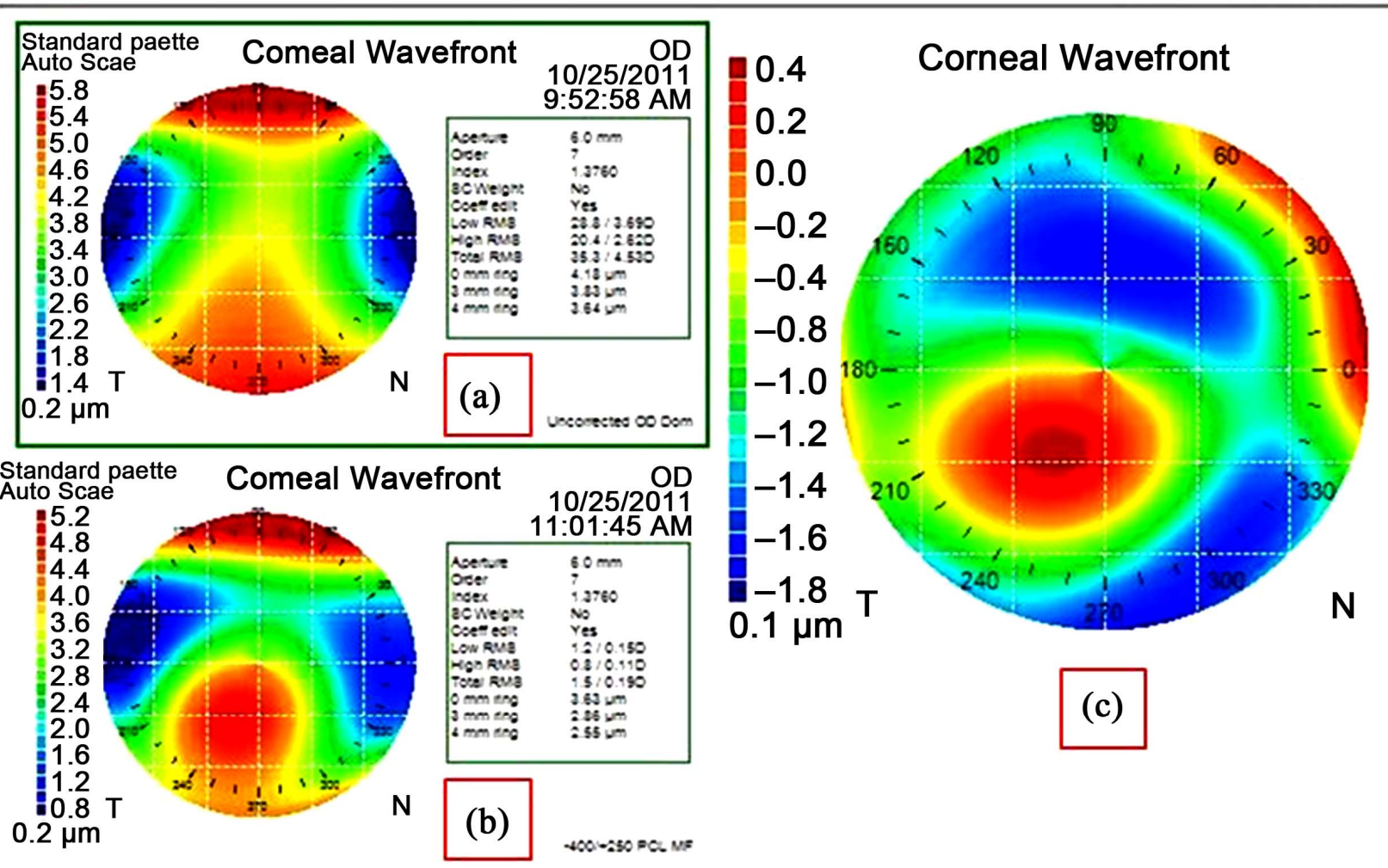

(C) 2011 CARL ZEISS MEDITEC ATLAS Revision 3.0.1.8

Figure 5. Color coded maps of corneal wavefront aberration profiles across a standardized 6 mm entrance pupil including 2nd order astigmatism and higher order aberrations up to the 7th Zernike order. (a) uncorrected cornea; (b) Proclear Multifocal D lens with +2.50 D Add power; (c) difference map (b minus a).

and mathematically expressed with Zernike coefficients. The nature of this measurement principle yields differences for the low order aberration $Z_{2}^{0}$ (sphere) between the reading of the naked cornea and the reading with the Proclear Multifocal D lens in place, since that's a desired change as given by the contact lens prescription. For that reason, $Z_{2}^{0}$ values have been removed from the topographical wavefront analysis. However of interest are all other wavefront errors, since they do also change due to wearing this specific type of contact lens and they cannot be selectively and purposeful corrected by ordering different contact lenses. Usually these are higher order aberrations, expressed as Zernike coefficients 3rd to 7th order.

In addition, one group of lower order aberrations, refractive cylinders, is also not compensated for with this specific type of multifocal soft contact lens, therefore the astigmatic Zernike coefficients $Z_{2}^{-2}$ and $Z_{2}^{2}$ have also been included in the analysis. For further evaluation, individual Zernike coefficients have been grouped per Zernike order and converted into RMS values. Clinical significance was assigned when a particular RMS value exceeded $0.10 \mu \mathrm{m}$ [30]. According to Fourier optics transformation, this is equivalent to a refractive error of $0.25 \mathrm{D}$, given the fact that the pupil diameter is $6 \mathrm{~mm}$. The transformation equation is given by

$$
M_{e}=4 \pi \sqrt{3} \frac{R M S}{r^{2}},
$$

where $r$ is the pupil radius in $\mathrm{mm}$.

\subsection{Statistical Analysis}

Statistical analysis was performed using the SPSS software package (v. 17.0; SPSS Inc., Chicago, IL, USA). Paired sample $t$-tests were conducted comparing the mean RMS value of the 2nd through 7th Zernike order for lenses with 4 different Add powers $+1.00 \mathrm{D},+1.50 \mathrm{D}$, $+2.00 \mathrm{D}$, and $+2.50 \mathrm{D}$ to the mean RMS value of 40 dominant eyes. For statistical purposes, a p value lower than 0.05 was considered statistically significant.

\section{Results}

The following 5 box-and-whisker diagrams (Figures 6 - 
10) display grouped RMS values of corneal wavefront aberrations with and without Proclear Multifocal D contact lenses in different Add powers. The bottom and top of each box are the lower and upper quartiles, respectively, and the line near the middle of the box is the median.

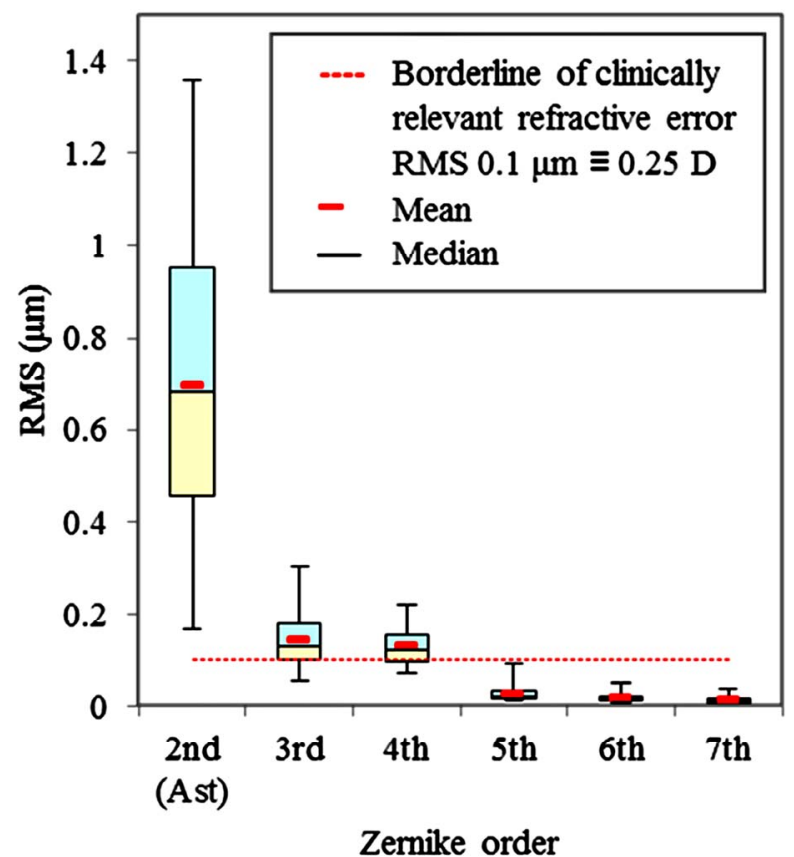

Figure 6. Box-and-whisker diagram of corneal wavefront aberrations expressed as grouped RMS values per Zernike order.

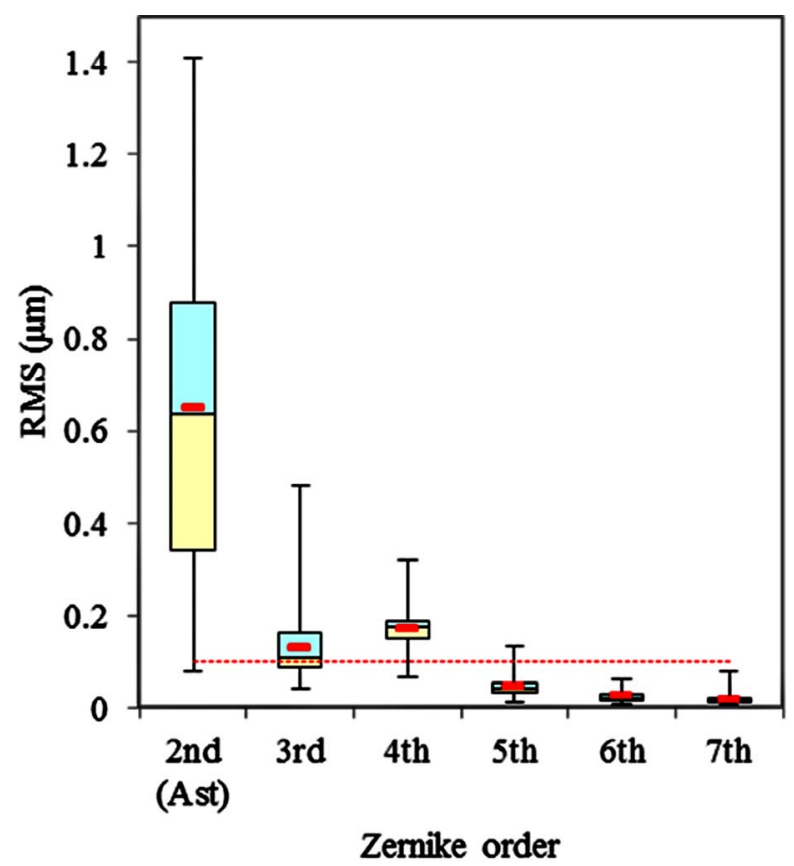

Figure 8. Proclear Multifocal D, +1.50 D Add power, boxand-whisker diagram, grouped RMS values of wavefront aberrations.
The small red line within the box is the mean. The ends of the whiskers represent the minimum and maximum of all RMS data. The horizontal dashed red line at $0.1 \mu \mathrm{m}$ represents the borderline of clinical significance. RMS values above this line equal refractive errors of $\geq 0.25 \mathrm{D}$.

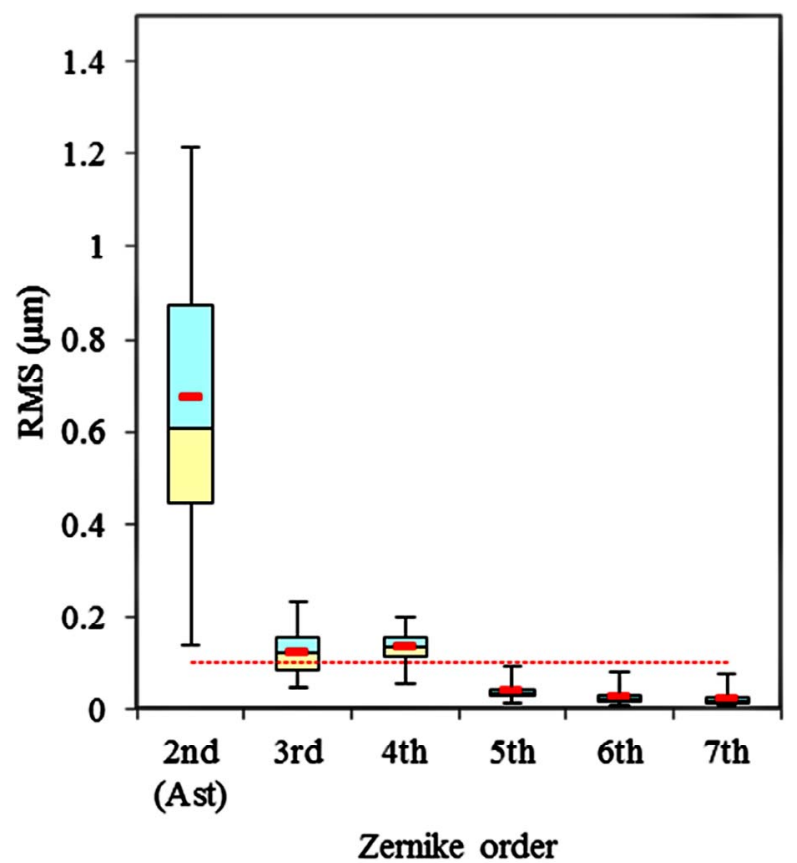

Figure 7. Proclear Multifocal D, +1.00 D Add power, boxand-whisker diagram, grouped RMS values of wavefront aberrations.

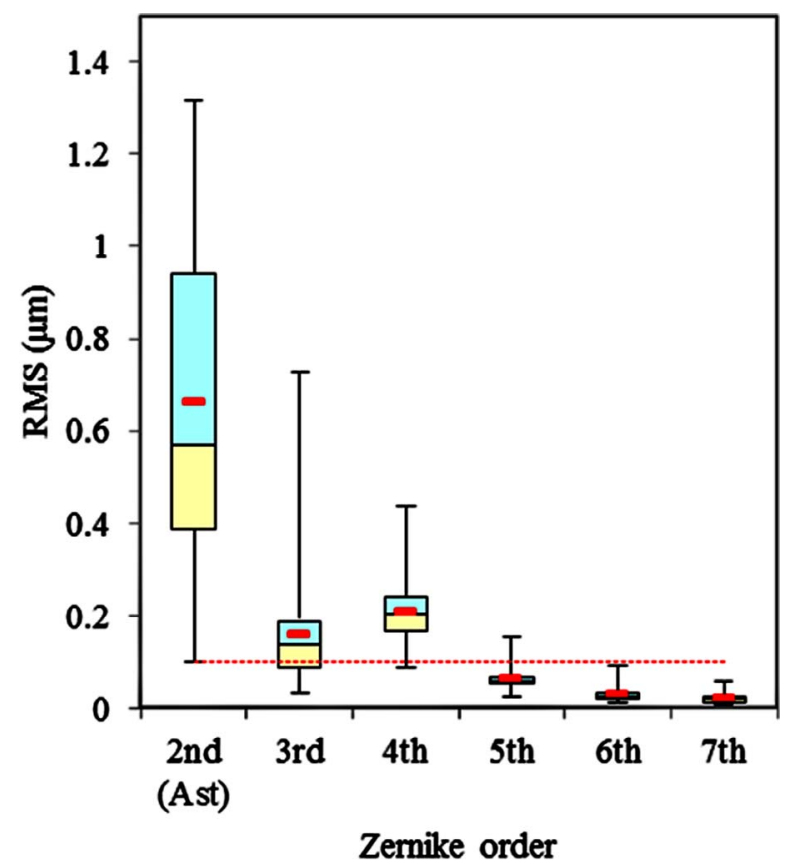

Figure 9. Proclear Multifocal D, +2.00 D Add power, boxand-whisker diagram, grouped RMS values of wavefront aberrations. 


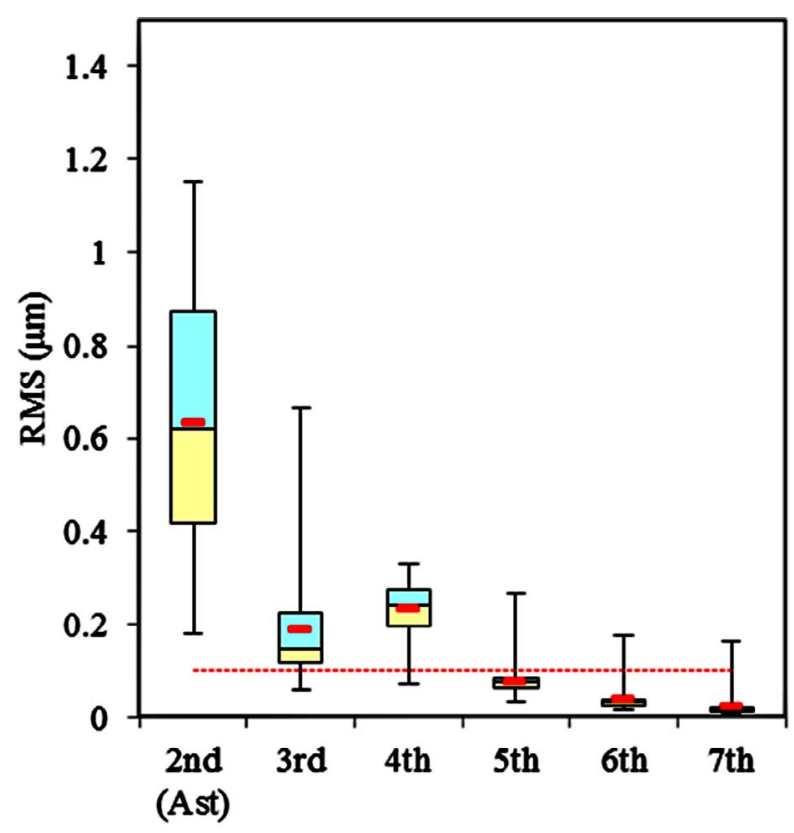

Zernike order

Figure 10. Proclear Multifocal D, +2.50 D Add power, boxand-whisker diagram, grouped RMS values of wavefront aberrations.

Table 1 summarizes RMS values of wavefront aberrations expressed as Zernike coefficients initially present for the patients' corneas and the mean difference and standard deviation of their changes with the different Add power groups. Clinically relevant aberrations and aberration changes were present for the initial corneal measurements and for all lens groups tested. Clinical relevance has been assigned for RMS values above 0.1 $\mu \mathrm{m}$ which is the refractive equivalent of $\geq 0.25 \mathrm{D}$.

Statistical significance is predominantly given for higher order aberrations and rises with increasing Add power. For the lens group with $+2.50 \mathrm{D}$ Add power, all aberration changes were statistically significant. In addition, clinically relevant wavefront aberrations in this group were 2nd Zernike order (astigmatism) with a RMS value of $0.63 \pm 0.29 \mu \mathrm{m}$, 4th Zernike order with a RMS value of $0.23 \pm 0.06 \mu \mathrm{m}$, and 3rd Zernike order with a RMS value of $0.18 \pm 0.12 \mu \mathrm{m}$. Notably lower order aberrations decreased, but all higher order aberrations increased. The highest increase was measured for 4th order aberrations. Interestingly this lens group has been suggested as the most effective for myopia progression control [31].

\section{Discussion}

Concentric multifocal center-distance soft contact lenses have been proposed as a treatment for children and young adults for the purpose of myopia progression control. In vivo, these lenses have the ability to mimic optical properties of corneas following successful Orthokeratology treatments, correcting refractive errors at the central retina and inducing myopic defocus at the retinal periphery. The advantages of using soft contact lenses are expected, immediate comfort and ease of fitting. Soft lenses typically have little to no awareness from the outset and the fitting parameters necessary are minimal simplifying the fitting process and initial lens selection. Since these lenses may compete with Orthokeratology for being selected as the system of choice in myopia progression control, it was of interest to see what their induced optical aberrations were and whether or not these data are equivalent to those reported for Orthokeratology.

In our study we investigated the change in corneal wavefront aberrations, especially astigmatism and higher order aberrations which occurred when Proclear Multifocal D lenses of different Add power groups were worn. With these lenses on the patients' eyes we did find substantial amounts of astigmatism and higher order wavefront aberrations for all tested lens groups. In general we observed that lenses with higher Add power values had larger changes in wavefront aberrations.

Statistically significant changes in aberrations, espe-

Table 1. RMS values of baseline corneal wavefront errors (Mean) and mean differences induced by Proclear Multifocal D lenses (MD) as well as their standard deviations (SD); statistical significance $(*)$ and clinical significance ( $\dagger$ ) have been assigned.

\begin{tabular}{cllllllllll}
\hline \multirow{2}{*}{$\begin{array}{c}\text { Zernike } \\
\text { order }\end{array}$} & Cornea & \multicolumn{4}{c}{$+1.00 \mathrm{D}$ Add } & \multicolumn{2}{l}{$+1.50 \mathrm{D}$ Add } & \multicolumn{2}{l}{$+2.00 \mathrm{D}$ Add } & \multicolumn{2}{c}{$+2.50 \mathrm{D}$ Add } \\
\cline { 2 - 11 } & Mean & SE & MD & SE & MD & SE & MD & SE & MD & SE \\
\hline 2nd(Ast) & $0.694^{\dagger}$ & 0.051 & $-0.022^{\dagger}$ & 0.021 & $-0.008^{\dagger}$ & 0.037 & $-0.035^{\dagger}$ & 0.021 & $-0.062^{* \dagger}$ & 0.026 \\
3rd & $0.139^{\dagger}$ & 0.009 & $-0.019^{* \dagger}$ & 0.009 & $-0.006^{\dagger}$ & 0.014 & $0.018^{\dagger}$ & 0.019 & $0.046^{* \dagger}$ & 0.019 \\
4th & $0.126^{\dagger}$ & 0.006 & $0.004^{\dagger}$ & 0.006 & $0.049^{* * *}+$ & 0.008 & $0.081^{* * *}$ & 0.008 & $0.106^{* * *+}$ & 0.008 \\
5th & 0.025 & 0.003 & $0.010^{* *}$ & 0.003 & $0.018^{* * *}$ & 0.004 & $0.034^{* * *}$ & 0.004 & $0.050^{* * *}$ & 0.005 \\
6th & 0.017 & 0.002 & $0.008^{* *}$ & 0.003 & $0.006^{* *}$ & 0.002 & $0.012^{* * *}$ & 0.003 & $0.017^{* * *}$ & 0.004 \\
7th & 0.012 & 0.001 & $0.007^{* *}$ & 0.002 & $0.004^{*}$ & 0.002 & $0.007^{* * *}$ & 0.002 & $0.009^{*}$ & 0.004 \\
\hline
\end{tabular}

MD: Mean Difference; SE: Standard Error of the Mean; Statistical significance: ${ }^{*} \mathrm{p}<0.05 ;{ }^{* *} \mathrm{p}<0.01 ;{ }^{* * *} \mathrm{p}<0.001$; Clinical significance: $†$. 
cially for higher order aberrations, were detected for all lenses. With all lenses the initial astigmatism decreased and most of the higher order aberrations increased. We further found that RMS values with lenses for 2nd (astigmatism), 3rd and 4th Zernike order remained in clinically significant amounts which was related to a wavefront error of $0.10 \mu \mathrm{m}$ or greater, the equivalent of $0.25 \mathrm{D}$. The highest aberration was astigmatism which was present for most patients initially, and although it was reduced with contact lenses, it remained the dominant aberration with all tested lenses. As anticipated, most lenses increased 4th order aberration significantly, thus underscoring the effectiveness of these lenses for myopia control.

In a recent study, Lopes-Ferreira et al. investigated central and peripheral states of objective refraction (low order aberrations) with Proclear Multifocal D lenses. They reported that only lenses with high Add power induced the amount of peripheral myopization necessary for effective myopia control [31].

With this same lens group (+2.50 D Add power), our study revealed the clinically significant wavefront aberrations to be 2nd order astigmatism (lower order aberration) with a RMS value of $0.63 \pm 0.29 \mu \mathrm{m}$, followed by higher order aberrations from the 4th Zernike order with a RMS value of $0.23 \pm 0.06 \mu \mathrm{m}$, and 3rd Zernike order with a RMS value of $0.18 \pm 0.12 \mu \mathrm{m}$. In direct comparison to wavefront aberrations of the naked cornea, lenses from this group decreased 2nd order astigmatism, but increased all higher order aberrations in a statistically significant manner.

Several authors report that Orthokeratology is a potential treatment system for myopia progression control [25, 26]. Multifocal soft contact lenses have been proposed for the same purpose $[21,22]$. One can assume that in this case wavefront aberrations would have a pattern similar to Orthokeratology. However, our findings with Proclear Multifocal D lenses differ from those reported by Joslin et al. for wavefront aberrations following Orthokeratology where astigmatism was almost eliminated and higher order aberrations were increased to much higher values. In their study, the resulting RMS values were $0.56 \pm 0.37$ $\mu \mathrm{m}$ for $3 \mathrm{rd}$ order aberrations, and $0.43 \pm 0.15 \mu \mathrm{m}$ for 4 th order aberrations [28]. These values are approximately 3 -fold higher for 3 rd order aberrations and almost 2-fold higher for 4th order aberrations in comparison to the Proclear Multifocal +2.50 D Add lenses, that being the lens group with the highest Add power and evidently highest aberrations analyzed in our study.

Therefore, in comparing lower and higher order wavefront aberrations, Proclear Multifocal D soft contact lenses and Orthokeratology are both seen as viable treatment systems for myopia progression control with conceptually related, but remarkably distinct, optical outcomes.
Further studies are necessary to determine whether or not the differences in the amount of aberrations as well as their selective variation and changes have clinical relevance for the course of myopia progression and myopia progression control.

\section{Acknowledgement}

The authors thank Mark Andre and Coopervision for providing the Proclear Multifocal D lenses used in this study, and Professor Jim Schwiegerling for providing the Zernike Pyramid image depicted in Figure 1.

\section{REFERENCES}

[1] S. M. Saw, J. Katz, O. D. Schein, S. J. Chew and T. K. Chan, "Epidemiology of Myopia," Epidemiologic Reviews, Vol. 18, No. 2, 1996, pp. 175-187.

[2] K. E. Lee, B. E. K. Klein, R. Klein and T. Y. Wong, "Changes in Refraction over 10 Years in an Adult Population: The Beaver Dam Eye Study," Investigative Ophthalmology \& Visual Science, Vol. 43, No. 8, 2002, pp. 2566-2571.

[3] L. L. K. Lin, Y. F. Shih, C. B. Tsai, et al., "Epidemiologic Study of Ocular Refraction among Schoolchildren in Taiwan in 1995," Optometry and Vision Science, Vol. 76, No. 5, 1999, pp. 275-281. doi:10.1097/00006324-199905000-00013

[4] R. D. Sperduto, D. Seigel, J. Roberts and M. Rowland, "Prevalence of Myopia in the United States," Archives of Ophthalmology, Vol. 101, No. 3, 1983, pp. 405-407. doi:10.1001/archopht.1983.01040010405011

[5] S. M. Saw, E. C. Shih-Yen, A. Koh and D. Tan, "Interventions to Retard Myopia Progression in Children: An Evidence-Based Update," Ophthalmology, Vol. 109, No. 3, 2002, pp. 415-421.

[6] K. E. Lee, B. E. Klein, R. Klein and T. Y. Wong, "Changes in Refraction over 10 Years in an Adult Population: The Beaver Dam Eye Study," Investigative Ophthalmology \& Visual Science, Vol. 43, No. 8, 2002, pp. 2566-2571.

[7] L. L. Lin, Y. F. Shih, C. B. Tsai, et al., "Epidemiologic Study of Ocular Refraction among Schoolchildren in Taiwan in 1995," Optometry and Vision Science, Vol. 76, No. 5, 1999, pp. 275-281. doi:10.1097/00006324-199905000-00013

[8] C. J. Hammond, H. Snieder, C. E. Gilbert and T. D. Spector, "Genes and Environment in Refractive Error: The Twin Eye Study," Investigative Ophthalmology \& Visual Science, Vol. 42, No. 6, 2001, pp. 1232-1236.

[9] I. Morgan and K. Rose, "How Genetic Is School Myopia?" Progress in Retinal and Eye Research, Vol. 24, No. 1, 2005, pp. 1-38. doi:10.1016/j.preteyeres.2004.06.004

[10] E. L. Smith, C. S. Kee, R. Ramamirtham, Y. QiaoGrider and L. F. Hung, "Peripheral Vision Can Influence Eye Growth and Refractive Development in Infant Monkeys," Investigative Ophthalmology \& Visual Sci- 
ence, Vol. 46, No. 11, 2005, pp. 3965-3972. doi:10.1167/iovs.05-0445

[11] C. E. Campbell, "A New Method for Describing the Aberrations of the Eye Using Zernike Polynomials," Optometry and Vision Science, Vol. 80, No. 1, 2003, pp. 79-83. doi:10.1097/00006324-200301000-00011

[12] D. A. Atchison, N. Pritchard, S. D. White and A. M. Griffiths, "Influence of Age on Peripheral Refraction," Vision Research, Vol. 45, No. 6, 2005, pp. 715-720. doi:10.1016/j.visres.2004.09.028

[13] D. A. Atchison, N. Pritchard, K. L. Schmid, D. H. Scott, C. E. Jones and J. M. Pope, "Shape of the Retinal Surface in Emmetropia and Myopia," Investigative Ophthalmology \& Visual Science, Vol. 46, No. 8, 2005, pp. 2698-2707. doi:10.1167/iovs.04-1506

[14] Z. Lin, A. Martinez, X. Chen, et al., "Peripheral Defocus with Single-Vision Spectacle Lenses in Myopic Children," Optometry and Vision Science, Vol. 87, No. 1, 2010, pp. 4-9. doi:10.1097/OPX.0b013e3181c078f1

[15] H. A. Swarbrick, "Orthokeratology (Corneal Refractive Therapy): What Is It and How Does It Work?" Eye Contact Lens, Vol. 30, No. 4, 2004, pp. 181-185.

[16] P. Sankaridurg, L. Donovan, S. Varnas, et al., "Spectacle Lenses Designed to Reduce Progression of Myopia: 12-Month Results," Optometry and Vision Science, Vol. 87, No. 9, 2010, pp. 631-641. doi:10.1097/OPX.0b013e3181ea19c7

[17] T. C. Tepelus, D. Vazquez, A. Seidemann, D. Uttenweiler and F. Schaeffel, "Effects of Lenses with different Power Profiles on Eye Shape in Chickens," Vision Research, Vol. 54, No. 1, 2012, pp. 12-19. doi:10.1016/j.visres.2011.11.014

[18] W. N. Charman, J. Mountford, D. A. Atchison and E. L. Markwell, "Peripheral Refraction in Orthokeratology Patients," Optometry and Vision Science, Vol. 83, No. 9, 2006, pp. 641-648. doi:10.1097/01.opx.0000232840.66716.af

[19] P. Kang and H. Swarbrick, "Peripheral Refraction in Myopic Children Wearing Orthokeratology and GasPermeable Lenses," Optometry and Vision Science, Vol. 88, No. 4, 2011, pp. 476-482. doi:10.1097/OPX.0b013e31820f16fb

[20] E. Kwok, B. Patel, S. Backhouse and J. R. Phillips, "Peripheral Refraction in High Myopia with Spherical Soft Contact Lenses," Optometry and Vision Science, Vol. 89, No. 3, 2012, pp. 263-270.

[21] T. A. Aller and C. Wildsoet, "Bifocal Soft Contact Lenses as a Possible Myopia Control Treatment: A Case Report Involving Identical Twins," Clinical and Expe- rimental Optometry, Vol. 91, No. 4, 2008, pp. 394- 399. doi:10.1111/j.1444-0938.2007.00230.x

[22] N. S. Anstice and J. R. Phillips, "Effect of Dual-Focus Soft Contact Lens Wear on Axial Myopia Progression in Children," Ophthalmology, Vol. 118, No. 6, 2011, pp. 1152-1161. doi:10.1016/j.ophtha.2010.10.035

[23] T.-T. Lee and P. Cho, "Discontinuation of Orthokeratology and Myopic Progression," Optometry \& Vision Science, Vol. 87, No. 12, 2010, pp. 1053-1056.

[24] P. Sankaridurg, B. Holden, E, Smith, et al., "Decrease in Rate of Myopia Progression with a Contact Lens Designed to Reduce Relative Peripheral Hyperopia: OneYear Results," Investigative Ophthalmology \& Visual Science, Vol. 52, No. 13, 2011, pp. 9362-9367. doi:10.1167/iovs.11-7260

[25] S. W. Cheung, P. Cho and D. Fan, "Asymmetrical Increase in Axial Length in the Two Eyes of a Monocular Orthokeratology Patient," Optometry \& Vision Science, Vol. 81, No. 9, 2004, pp. 653-656. doi:10.1097/01.opx.0000144742.57847.b1

[26] T. Kakita, T. Hiraoka and T. Oshika, "Influence of Overnight Orthokeratology on Axial Elongation in Childhood Myopia," Investigative Ophthalmology \& Visual Science, Vol. 52, No. 5, 2011, pp. 2170-2174. doi:10.1167/iovs.10-5485

[27] S. Vitale, L. Ellwein, M. F. Cotch, F. L. Ferris and R. Sperduto, "Prevalence of Refractive Error in the United States (1999-2004)," Archives of Ophthalmology, Vol. 126, No. 8, 2008, pp. 1111-1119.

[28] C. E. Joslin, S. M. Wu, T. T. McMahon and M. Shahidi, "Higher-Order Wavefront Aberrations in Corneal Refractive Therapy," Optometry and Vision Science, Vol. 80 , No. 12, 2003, pp. 805-811. doi:10.1097/00006324-200312000-00010

[29] W. S. Chui and P. Cho, "A Comparative Study of the Performance of Different Corneal Topographers on Children with Respect to Orthokeratology Practice," Optometry \& Vision Science, Vol. 82, No. 5, 2005, pp. 420-427. doi:10.1097/01.OPX.0000162642.24885.71

[30] C. McAlinden, J. E. Moore, V. E. McGilligan, T. C. B. Moore, "Spherical Aberration and Higher Order Aberrations with Balafilcon A (Pure Vision) and Comfilcon A (Biofinity)," Graefes Archive for Clinical and Experimental Ophthalmology, Vol. 249, No. 4, 2011, pp. 607612. doi:10.1007/s00417-010-1476-9

[31] D. Lopes-Ferreira, C. Ribeiro, R. Maia, et al., "Peripheral Myopization Using a Dominant Design Multifocal Contact Lens," Journal of Optometry, Vol. 4, No. 1, 2011, pp. 14-21. doi:10.1016/S1888-4296(11)70035-8 